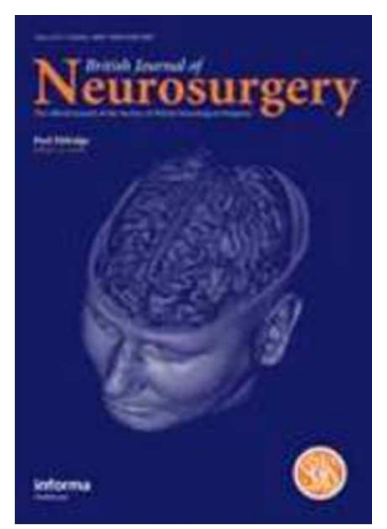

\title{
Medical student perceptions of clinical neurosurgery teaching in an undergraduate medical school curriculum
}

\begin{tabular}{|r|l|}
\hline Journal: & British Journal of Neurosurgery \\
\hline Manuscript ID & CBJN-2016-0297.R2 \\
\hline Manuscript Type: & Original Article \\
\hline Date Submitted by the Author: & n/a \\
\hline Complete List of Authors: & $\begin{array}{l}\text { Knight, James; St George's University of London } \\
\text { Stroud, Lauren; St George's, University of London } \\
\text { Geyton, Thomas; St George's, University of London } \\
\text { Stead, Anthony; St George's, University of London, Institute of Medical \& } \\
\text { Biomedical Education } \\
\text { Cock, Hannah; St George's, University of London, Institute of Medical \& } \\
\text { Biomedical Education; St George's University Hospitals NHS Foundation } \\
\text { Trust, Atkinson Morley Regional Neuroscience Centre }\end{array}$ \\
\hline Keywords: & $\begin{array}{l}\text { Basic neurosciences training, Neuroanatomy, Generic training, Operative } \\
\text { experience, Neurosurgery }\end{array}$ \\
\hline
\end{tabular}

\section{SCHOLARONE ${ }^{\text {Tw }}$}

Manuscripts 


\title{
Medical student perceptions of clinical neurosurgery teaching in an undergraduate medical school curriculum
}

Authors: Knight, James ${ }^{1}$; Stroud, Lauren ${ }^{1}$; Geyton, Thomas ${ }^{1}$; Stead, Anthony ${ }^{1}$; Cock, Hannah $\mathrm{R}^{2,3}$.

${ }^{1}$ Medical Students, St George's University of London, Cranmer Terrace, London SW17 ORE ${ }^{2}$ Institute of Medical and Biomedical Education, St George's University of London, Cranmer Terrace, London SW17 ORE

${ }^{3}$ Atkinson Morley Regional Neuroscience Centre, St George's University Hospitals NHS Foundation Trust, Blackshaw Road, London SW17 0QT

Corresponding author: Professor Hannah Cock

Correspondence address: Institute of Medical and Biomedical Education, St George's University of London, Cranmer Terrace, London SW17 ORE

Email: hannahrc@sgul.ac.uk

Phone: 02087252002

Guarantor/Senior author: Professor Hannah Cock

Key words: education, neurophobia, neurosurgery, Undergraduate, Neuroanatomy, perceived learning

Abstract: 281 words

Word count: 1750

References: 13

\begin{abstract}
Aim: The aim of this study was to evaluate undergraduate medical student perceptions as to the value of different types of neurosurgical teaching to their general neuroscience education, delivered in the penultimate year of a UK medical school.
\end{abstract}


Methods: We surveyed penultimate-year medical students at St George's Hospital Medical School, University of London (SGUL), who were undertaking their clinical neuroscience attachment from August 2014 to July 2015. A questionnaire comprising closed Likert scale questions and an open question inviting participants to comment freely was used to assess student perception about the value of Neurosurgical sessions within their overall neuroscience education.

Results: Of the 316 students in the year we surveyed 247 (78.2\%), of whom 201 responded (response rate $81.4 \%$ ). On average, $82.8 \%$ of students either agreed or strongly agreed that neurosurgical teaching sessions made a valuable contribution to their learning. In particular, lectures by neurosurgeons, clinical teaching on the Glasgow Coma Scale in neuro-ITU, bedside teaching and neurosurgical clinics were considered the most beneficial. The majority of students felt the sessions improved their understanding of neurological examination, signs, and 'redflags'. The sessions were also beneficial for learning neuro-imaging and understanding of neurosurgical emergencies. Over two thirds felt that theatre sessions were beneficial, significantly more so amongst students invited to 'scrub-in'.

Conclusion: Students rated neurosurgical sessions highly and valued the contribution they made not only to their learning of neurosurgical conditions and emergencies, but also to their learning of general neurology and clinical neurosciences overall. Student perceived learning from theatre sessions was significantly correlated with whether or not the student had been invited to 'scrub-in'. Expert neurosurgical teaching can make a valuable, and arguably essential contribution to the undergraduate medical curriculum.

\section{Introduction}


Advances in medical knowledge and technology are making it more difficult to produce graduates who are well rounded in modern medicine and trained to deliver safe and effective patient care ${ }^{1,2}$, with competition for space in the undergraduate medical curriculum increasingly pressured. This has included a drive towards a more primary care and generalist approach, such that teaching of modern 'tertiary' specialties some argue is being pushed out of the syllabus $^{3}$. That it is important for modern clinicians to be trained to deal with common or urgent conditions that affect the general population remains explicit in training guidance as it has for some years ${ }^{4}$. Neurosurgical conditions that fall into these groups include intracranial haemorrhage, head injury, spinal disease and raised intracranial pressure. Whilst the initial investigation, diagnosis and management of such conditions may often be carried out by nonspecialists (e.g. general practitioners, emergency and general physicians) failure to involve specialists has been shown to run the risk of inadvertently perpetuating common errors ${ }^{5}$. Surveys both in the $\mathrm{USA}^{6}$ and recently in the $\mathrm{UK}^{3}$ support that whilst neurosurgical content is covered in most curricula, in many medical schools core neurosurgical teaching is delivered via non-specialist clinicians who may be under-equipped to optimally manage the early stages of neurosurgical conditions. This is of particular concern for neurosurgical emergencies ${ }^{7}$. There is also some evidence that including neurosurgery as part of the undergraduate curriculum is of benefit to broader clinical neuroscience learning ${ }^{8}$. The student perspective on this has not previously been explored in the literature.

We set out to evaluate undergraduate medical student perceptions as to the value of different types of neurosurgical teaching to both key neurosurgical topics and their general neuroscience education.

\section{Materials and methods}

Study population 
Consecutive cohorts of penultimate-year medical students who had just completed their clinical neuroscience attachment at the associated Regional Neuroscience Centre between August 2014 and July 2015 were invited to participate in the study. Following review of an information sheet provided at their final-sign off meeting, and verbal consent, a face-face structured interview and questionnaire were completed.

The clinical neuroscience attachment is a 5-week placement covering neurology, stroke, neurosurgery and neurorehabilitation. Exposure to all components is compulsory, and includes structured lectures, clinical demonstrations, and small group teaching over the 5-weeks alongside time on the wards and clinics. Students must attend a specified minimum of 4 general neurology and 2 specialist clinics, on call shifts for neurosurgery and for neurology/stroke, a 2 day neurorehablitation placement and one neurosurgical theatre session, but have some flexibility and choice around which additional sub-specialist clinics to attend (including neurosurgery). Approximately $15 \%$ of teaching hours (5.4hours/week) delivered to Penultimateyear students within clinical neurosciences are from neurosurgery, who also receive a commensurate remuneration via SIFT (Service Increment For Training) funding, mapped on an annual basis through a departmental teaching diary.

\section{Evaluation questionnaire}

The questionnaire (appendix 1) included student demographics and closed questions with a range of responses using a Likert scale of strongly agree to strongly disagree. An open question was included at the end to invite participants to comment freely. Question matrices were used to assess to what extent neurosurgical sessions contributed to the university's specified clinical neuroscience learning objectives. The final question matrix considered the compulsory theatre session students attended.

\section{Results}


Response rate and Demographics

Of the 316 students in the year $247(78.2 \%)$ were invited to participate in the survey of which 201 responded giving a response rate of $81.4 \%$. Timetabling conflicts meant not all cohorts/students could be invited. Penultimate year medical students were made up of three groups; those studying a traditional 5-year undergraduate course $(54.7 \%, n=110)$, graduate students studying a shorter 4-year degree $(37.8 \%, n=76)$, and a small number of biomedical transfer students who enter the course during the preceding academic year $(7.5 \%, \mathrm{n}=15)$, directly after completing a BSc in Biomedical Sciences. Just over half $(54.2 \%, n=110)$ were female, and one student opted not to disclose their gender. With respect to career aspirations, $45(22.4 \%)$ agreed or strongly agreed they were considering a career in neurosurgery, 78 $(38.8 \%)$ any surgical specialty, and 66 (32.8\%) neurology. Subgroup analysis showed significantly more graduate students $(26 / 76,34.2 \%)$ were considering a career in neurosurgery than undergraduates $(19 / 110,17.3 \%$. p<0.01 Fishers Exact), or biomedical science transfer students $(0 / 15)$

\section{Educational outcomes}

With respect to the learning objective categories defined in the university curriculum as neurosurgical [Table 1], on average $82.8 \%$ of students either agreed or strongly agreed that the neurosurgical sessions they attended contributed to their learning, with only $8.5 \%$ disagreeing to any extent. In particular, lectures by neurosurgeons, a practical GCS teaching session in the neuro intensive care unit, bedside teaching and neurosurgical clinics were considered the most beneficial. A breakdown with respect to specific educational needs is shown in Table 2, illustrating the broad value of neurosurgical teaching.

Over two thirds $(67.7 \%, \mathrm{n}=136)$ of students agreed or strongly agreed that attending at least one neurosurgical theatre session was beneficial, while $22.9 \% \quad(n=46)$ disagreed or strongly disagreed. The majority of students enjoyed their theatre sessions, with $78.6 \%(n=158)$ 
feeling welcome and $67.2 \%(n=135)$ agreeing that surgeons were willing to teach. Only $16.1 \%$ of students $(n=33)$ scrubbed in, but those who did were significantly more likely to report this was beneficial for their learning (Figure 1)

\section{Discussion}

Our results show that medical students valued the neurosurgical component of their five week placement. Though neurosurgery is a tertiary specialty, all doctors need to be able to investigate and instigate the initial management of certain key conditions. This study found that neurosurgical placements were strongly beneficial not only to perceived overall learning of these conditions, but also of value more broadly to generic competencies relevant to neurological disorders recommended as core to the undergraduate curriculum ${ }^{9}$.

Furthermore, whilst few medical students and junior doctors will pursue neurosurgery as a career, all neurosurgical conditions initially present to non-neurosurgeons ${ }^{3}$. Thus, there is a particular need for these clinicians to be able to promptly recognise and initially manage these conditions, particularly with respect to neurosurgical emergencies. Neurosurgery is thus a vital part of medical school curricula ${ }^{3,7}$. Ralph et al $^{7}$ further argues medical students need to be taught by physicians who regularly see neurosurgical problems and are thus able to "impart a palpable sense of their presentation and impact upon the patient".

\section{Neurophobia}

There is a significant amount of literature on the topic of 'neurophobia', a term coined by Ralph in $1994^{10}$ to describe the syndrome of perceived difficulties medical students and junior doctors have with neurology and clinical neuroscience. A national UK medical student survey ${ }^{11}$ in $2014(n=2877)$ found that neurophobia was still prominent, with neurology rated as the most difficult, and associated with the lowest confidence in drawing up a differential diagnosis compared with cardiology, respiratory medicine, gastroenterology, geriatrics, rheumatology and 
endocrinology. Similarly, in a series of surveys ${ }^{12}$ on 186 London medical students, 100 junior doctors attending courses for MRCP examinations and 59 General Practitioners, neurology was consistently rated as the most difficult of these seven medical specialties, with the lowest selfratings for clinical knowledge and clinical confidence, despite high interest. This was most commonly attributed to poor teaching, trouble with neuroanatomy, and with clinical examination. Alongside neurosurgical sessions being beneficial to the learning of neurosurgical topics, our study found that students also valued the contribution neurosurgical sessions gave to their general neurological training including neurological examination, recognizing physical signs and red flags, and neuroimaging. This whilst we did not ask a direct question about confidence/feeling equipped, that the majority of students felt their educational needs were helped by neurosurgical teaching supports that the inclusion of neurosurgery in the undergraduate curriculum could reduce neurophobia and improve neurological skills. This is also supported by an American observational non-randomised comparative study ${ }^{5}$, in which students who had experienced a neuroscience placement which included a neurosurgery block outperformed those who had a standard neurology block without, in the neuroscience aspects of end of year exams

\section{Theatre}

For our cohort of students it was compulsory to attend at least one theatre session, which the majority of students felt was beneficial, especially those who scrubbed in. One previous small study conducted at Queen's University Belfast in $2014^{1}$, which surveyed 22 medical students who attended neurosurgical theatre as part of their placements, similarly reported that $68 \%(n=15)$ felt that their educational needs were met. Of note, only one of these students was invited to scrub in. We cannot exclude that the significant relationship between scrubbing in and learning is an association rather than causal. However clearly scrubbing in is essential for close proximity to the macroscopic aspects of the procedure, and if there are 
difficulties with seeing the surgery then its contribution to the learning of anatomy is likely to be poor. Thus we consider our study strongly supports the benefits of scrubbing in for student learning. Why the once traditional practice of students scrubbing-in appears to have waned over time $^{1}$ has not been explored, and is a potential topic for further research.

\section{On-call session}

. Sessions with the on-call neurosurgical registrar gave an opportunity for students to shadow one-to-one with a neurosurgeon who was taking referrals and seeing acute patients. The results, along with additional individual comments, showed this to be a highly valued session. Students felt they had good exposure to acute patients and witnessed presentations, examination findings and imaging being used in real-time clinical scenarios and decision making.

\section{Limitations}

Although there is a high response rate, not all students chose to participate in this study, and we cannot exclude a selection bias of participants influencing results. This is also a single centre study conducted across one academic year, the results of which may not be generalizable to other centres or in the longer term. Finally, we have assessed only perceived learning, and did not set out to measure actual performance, nor in the longer term improved patient outcomes as part of this study. Nonetheless, student satisfaction is an increasingly important metric in higher education. Furthermore interventions that improve student satisfaction and perception of learning commonly correlate with improved outcomes, and evidence supports that medical students, particularly later in their training as in this case, are at least moderately able to self-assess performance ${ }^{13}$.

\section{Conclusions}


Students valued the contribution of teaching from neurosurgeons highly, including theatres in particular where they were invited to scrub-in; neurosurgical teaching benefit not only to perceived learning about neurosurgical conditions and emergencies, but also with respect to their learning of general neurology and clinical neurosciences overall.

\section{Acknowledgments \\ Particular thanks to Sheree Manser (Undergraduate teaching administrator) for her logistical help and support.}

\section{Authorship:}

JK conceived and designed the study. HRC supervised the project. All authors contributed to the development of the survey, and JK, LS, AS and TG interviewed students one-to-one and collated data; JK, LS and HRC contributed to the analysis and interpretation of data. JK wrote the first draft, with critical review by all authors throughout, and substantial amendments and response to reviewers by JK and HRC. All authors provided approval of the final manuscript.

\section{References}

1. Flannery T, Gormley G. Evaluation of the contribution of theatre attendance to medical undergraduate neuroscience teaching - A pilot study. British Journal of Neurosurgery. 2014;28(5):680-4.

2. Stern WE. A statement concerning neurosurgery in the undergraduate medical curriculum. Surgical Neurology. 1990;33(1):5-6.

3. Whitehouse KJ, Moore AJ. Undergraduate teaching of neurosurgery - what is the current practice in the UK and is there a need for improvement? British Journal of Neurosurgery. 2015;29(6):753-7.

4. GMC. Outcomes for Graduates. London, UK: General Medical Council; 2016.

5. Corbett EC, Innes DJ, Resnick DK. Undergraduate Neuroscience Education. Journal of Neurosurgery. 2000;92(4):738-9.

6. Daniel K. Resnick. Neuroscience education of undergraduate medical students. Part I: role of neurosurgeons as educators. Journal of Neurosurgery. 2000;92(4):637-41.

7. Lehman RAW. Will future medical students be taught neurosurgery? Surgical Neurology. 1993;40(1):5-6.

8. Daniel K. Resnick, Lincoln F. Ramirez. Neuroscience education of undergraduate medical students. Part II: outcome improvement. Journal of Neurosurgery. 2000;92(4):642-5.

9. $\quad A B N$. Learning about Neurological Disorders: Recommendations for UK Medical Undergraduate Education. London, UK: Association of British Neurologists, 2015. 
10. Jozefowicz RF. Neurophobia: The fear of neurology among medical students. Archives of Neurology. 1994;51(4):328-9.

11. Pakpoor J, Handel AE, Disanto G, Davenport RJ, Giovannoni G, Ramagopalan SV. National survey of UK medical students on the perception of neurology. BMC medical education. 2014;14:225.

12. Schon F, Hart P, Fernandez C. Is clinical neurology really so difficult? Journal of Neurology Neurosurgery and Psychiatry. 2002;72(5):557-9.

13. Blanch-Hartigan D. Medical students' self-assessment of performance: Results from three meta-analyses. Patient Education and Counseling. 2011;84(1):3-9. 
1

2

3

4

5

6

7

8

9

10

Medical student perceptions of clinical neurosurgery teaching in an undergraduate medical school curriculum

Figure 1: Scrubbing-in benefits overall learning and neuroanatomy in particular

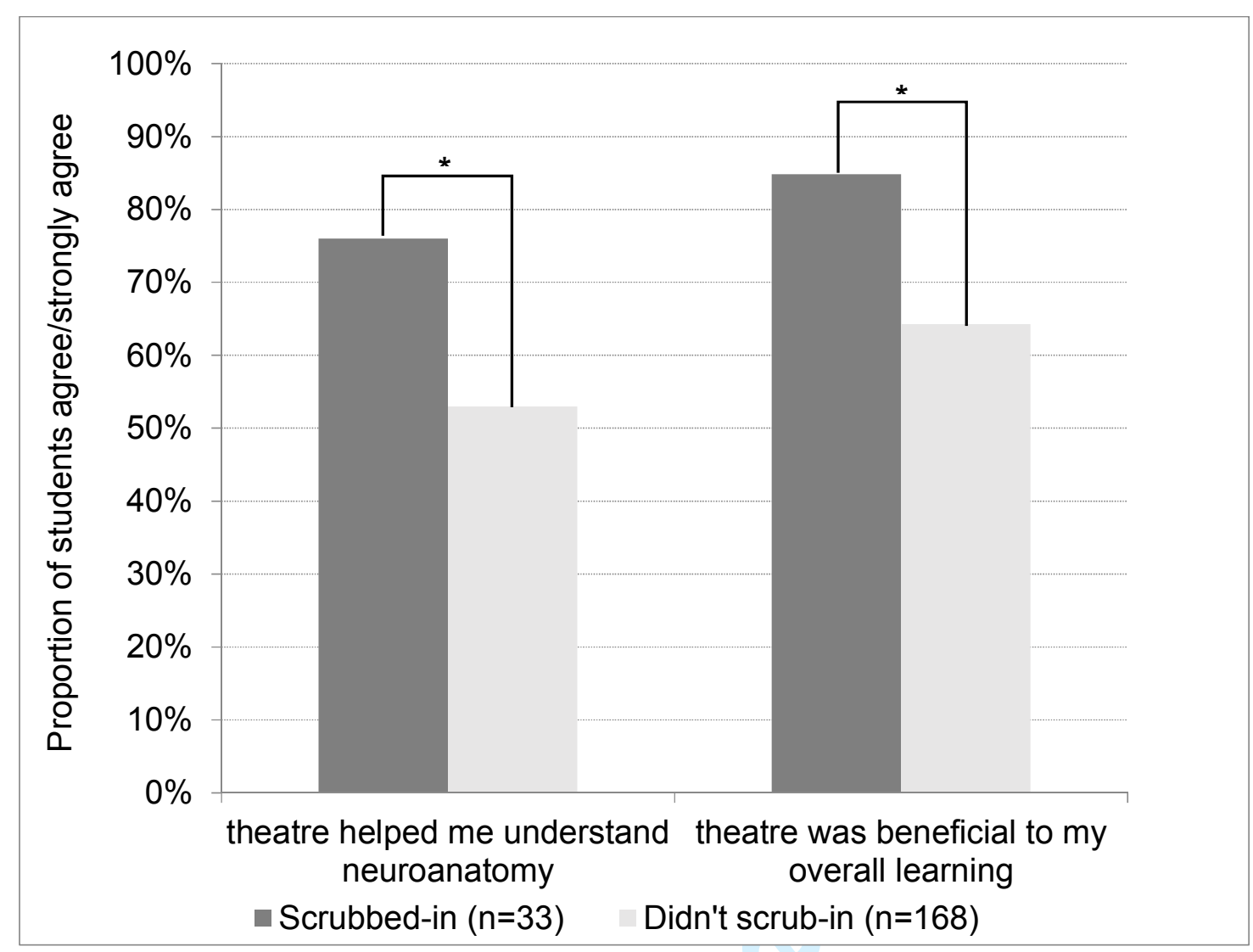

Proportion of students reporting that neurosurgical theatre attendance benefitted their learning with respect to neuro-anatomy, or overall ( ${ }^{*} p<0.05$ Fishers Exact) 


\section{An analysis of the value of clinical neurosurgery sessions in the undergraduate medical school curriculum.}

TABLE 1: Participant response to the statement 'With the learning objectives in mind, please indicate whether the following neurosurgical teaching sessions contributed to your learning'.

\begin{tabular}{|l|l|l|}
\hline Activity & $\begin{array}{l}\text { Agree or strongly agree } \\
\%(n)\end{array}$ & $\begin{array}{l}\text { Disagree or strongly } \\
\text { disagree } \%(n)\end{array}$ \\
\hline Theatre & $67.7(136)$ & $22.9(46)$ \\
\hline Neurosurgical on-call* & $79.6(160)$ & $10.0(20)$ \\
\hline Clinic (62 did not attend) & $82.0(114)$ & $5.8(8)$ \\
\hline Lectures (by neurosurgeons) & $90.6(182)$ & $1.5(3)$ \\
\hline ICU-GCS** & $91.1(183)$ & $3.5(7)$ \\
\hline Bedside teaching & $85.6(172)$ & $7.5(15)$ \\
\hline Average & $\mathbf{8 2 . 8}$ & $\mathbf{8 . 5}$ \\
\hline
\end{tabular}

The university curriculum includes specific neurosurgery learning objectives in relation to the diagnosis and initial management of 1) head injury 2) intracranial haemorrhage 3) spinal cord compression 4) spinal degenerative disorders 5) tumours of the nervous system and raised intracranial pressure. *Neurosurgical on-call: were evening sessions with the on-call registrar or SHO. **ICU-GCS: guided assessment of GCS on neuro-ICU patients. Remaining participants gave a neutral response in all instances (neither agree or disagree). 
An analysis of the value of clinical neurosurgery sessions in the undergraduate medical school curriculum.

TABLE 2: Participant response to the statement 'Neurosurgical sessions helped me with the below educational needs'.

\begin{tabular}{|l|l|l|}
\hline Educational need & $\begin{array}{l}\text { Agree or strongly agree } \\
\%(n)\end{array}$ & $\begin{array}{l}\text { Disagree or strongly } \\
\text { disagree \% }(n)\end{array}$ \\
\hline Neuro-exam & $72.6(146)$ & $14.4(29)$ \\
\hline Neuro-signs & $89.0(179)$ & $3.5(7)$ \\
\hline Neuroimaging & $83.0(167)$ & $5.0(10)$ \\
\hline Patient journeys & $61.2(123)$ & $7.5(15)$ \\
\hline Red-flags & $76.1(153)$ & $8.0(16)$ \\
\hline Emergencies & $69.7(140)$ & $9.5(19)$ \\
\hline Average & $75.1(151)$ & $8.0(16)$ \\
\hline
\end{tabular}




\section{Medical student perceptions of clinical neurosurgery teaching in an undergraduate medical school curriculum}

Appendix 1. Questionnaire

1. Which course are you on?
a. Graduate entry MBBS 4 year
b. Biomedical transfer MBBS 5 year
c. Undergraduate MBBS 5 year

2. What is your age?
a. $20-24$
b. $25-20$
c. $30-34$
d. $35-39$
e. $40+$

3. What is your gender?
a. Male
b. Female
c. Prefer not to say

4. Please rate the statements below (strongly disagree/disagree/neither disagree nor agree/agree/strongly agree)
a. I am considering neurology as a career
b. I am considering neurosurgery as a career
c. I am considering surgery as a career

5. The neurosurgery learning objectives included in the clinical neuroscience curriculum provided by the university covered these key areas:
I. Head injury
II. Intracranial haemorrhage
III. Spinal cord compression
IV. Spinal cord degeneration
V. CNS tumours and intracranial pressure

With the above learning objectives in mind, please rate how these neurosurgical sessions contributed to your learning? (strongly disagree/disagree/neither disagree nor agree/agree/strongly agree)
a. Theatre
b. Neurosurgical on call
c. Neurosurgical Clinic(s)
d. Neurosurgical lectures
e. Neuro ICU GCS session
f. Neurosurgical bedside teaching

6. Neurosurgical sessions helped me with the below educational needs: (strongly disagree/disagree/neither disagree nor agree/agree/strongly agree)
a. Neurological examination and technique
b. Spotting or understanding neurological signs
c. Interpreting basic neuro-imaging
d. Neurosurgical patient journeys
e. Understanding neurosurgical red-flags
f. Diagnosing emergencies and knowing when to refer to neurosurgeons (e.g. in A\&E)

7. Did you srub-in while in theatre ?
a. Yes
b. No 
Medical student perceptions of clinical neurosurgery teaching in an undergraduate medical school curriculum

Appendix 1. Questionnaire

8. With respect to neurosurgical theatre sessions, please rate the following statements (strongly disagree/disagree/neither disagree nor agree/agree/strongly agree)
a. It helped me understand neuroanatomy
b. Surgeons were willing to teach
c. I felt welcome in theatre
d. It was important to know the patients prior to the operation
e. I made a point of introducing myself to the surgical team

9. Can you recommend any improvements to the neurosurgical theatre sessions? (free text) 